\title{
A Importância da Explicitação da Correspondência Um para \\ Muitos na Resolução de Problemas de Estrutura Multiplicativa
}

\author{
The Importance of Expliciting the One-to-many Correspondence in Solving \\ Problems of Multiplicative Structure
}

\author{
Alina Galvão Spinillo * \\ ORCID iD 0000-0002-6113-4454 \\ Síntria Labres Lautert ${ }^{* *}$ \\ ORCID iD 0000-0002-7732-0999 \\ Ernani Martins dos Santos *** \\ ORCID iD 0000-0002-3824-986X
}

\begin{abstract}
Resumo
Diversos aspectos influenciam a resolução de problemas multiplicativos por crianças, como as características numéricas das tarefas e os suportes de representação disponibilizados durante os procedimentos de resolução. $\mathrm{O}$ presente estudo investiga o papel desempenhado por um outro aspecto que é constitutivo do raciocínio multiplicativo: a correspondência um para muitos. Esta correspondência pode estar implícita ou explicitamente mencionada no enunciado dos problemas. Neste estudo examinou-se a possibilidade de que o nível de explicitação da relação um para muitos seria fator importante na resolução de problemas multiplicativos, e que quanto mais explícitas fossem essas relações, melhor seria o desempenho das crianças. Para isso, problemas de multiplicação e de divisão foram solucionados por crianças do $3^{\circ}$ e $5^{\circ}$ ano do Ensino Fundamental. Os problemas apresentavam diferentes níveis de explicitação da correspondência uma para muitos. Observou-se que o desempenho era melhor nos problemas em que a correspondência uma para muitos era explicitamente mencionada no enunciado do que em problemas em que esta relação estava implícita. Isso foi observado em ambos os anos escolares. Os resultados evidenciam o papel facilitador que a explicitação da correspondência um para muitos desempenha na resolução de problemas de estrutura multiplicativa. Implicações teóricas e educacionais são discutidas.
\end{abstract}

Palavras-chave: Resolução de Problemas. Estrutura Multiplicativa. Correspondência Um para Muitos. Crianças.

\begin{abstract}
The way in which children solve multiplicative problems is influenced by several aspects, such as the numerical characteristics of the tasks and the representation support that is available during the solution procedure. The

\footnotetext{
* Doutora em Psicologia do Desenvolvimento pela University of Oxford, Inglaterra. Professora Titular do Programa de Pós-Graduação em Psicologia Cognitiva da Universidade Federal de Pernambuco (UFPE), Recife, Pernambuco, Brasil. E-mail: alinaspinillo@ hotmail.com.

** Doutora em Psicologia Cognitiva pela Universidade Federal de Pernambuco (UFPE). Professora Associada IV Departamento de Psicologia e da Pós-graduação em Psicologia Cognitiva da Universidade Federal de Pernambuco (UFPE), Recife, Pernambuco, Brasil. E-mail: sintria.lautert@ufpe.br.

*** Doutor em Psicologia Cognitiva pela Universidade Federal de Pernambuco (UFPE). Professor Adjunto e PróReitor de Graduação da Universidade de Pernambuco (UPE), atuando no Programa de Pós-Graduação em Formação de Professores e Práticas Interdisciplinares (campus Petrolina) e na Graduação em Matemática (campus Mata Norte), Recife, Pernambuco, Brasil. E-mail: ernani.santos@upe.br.
} 
present study investigates the role played by another aspect of multiplicative reasoning: the one-to-many correspondence. This correspondence may be implicitly or explicitly mentioned in the wording of the problem. This study examined whether the degree of explicitness of the one-to-many relationship is an important factor in the solution of multiplicative problems, and whether the more explicit this correspondence is, the better the child's performance. With that in mind, 3rd and 5th grades elementary school students were asked to solve multiplication and division problems. In the wording of the problems, the one-to-many correspondence was presented in different degrees of explicitness. It was found that performance was better in problems in which the one-to-many correspondence was explicitly mentioned than in those in which this relationship was implicit, a fact observed in both school grades. Results show the facilitating role played by the explicitness of the one-to-many correspondence in the solution of problems of multiplicative structure. Theoretical and educational implications are discussed.

Keywords: Problem solving. Multiplicative structure. Correspondence one-to-many. Children.

\section{Introdução}

Um dos aspectos de interesse entre pesquisadores e educadores no campo da Educação Matemática tem sido identificar que situações seriam mais favoráveis de modo a permitir que crianças resolvessem com sucesso problemas de estrutura multiplicativa, uma vez que os conceitos inseridos neste campo são desenvolvidos por um longo período durante a vida escolar. Diversos aspectos são considerados facilitadores do raciocínio das crianças na resolução de problemas deste tipo, sendo os mais frequentemente apontados na literatura as características numéricas das tarefas (quantificação de grandezas discretas ou contínuas ${ }^{1}$, números naturais ou racionais em sua forma fracionária ou decimal) e a natureza do suporte de representação disponibilizado durante o processo de resolução (material concreto, lápis e papel, diagrama, recursos digitais, calculadora). O presente estudo examina o papel desempenhado por um outro aspecto que está subjacente e é constitutivo do raciocínio multiplicativo: a relação um para muitos. Esta relação pode estar implícita ou explícita no enunciado dos problemas, o que pode ter um impacto sobre o desempenho das crianças em problemas de natureza multiplicativa, como discutido adiante e examinado nesta investigação.

Diversos autores enfatizam o efeito que as características numéricas da tarefa têm no desempenho e nas estratégias utilizadas na resolução de problemas. As quantidades discretas ou contínuas, números inteiros ou fracionários, números inteiros pequenos (um ou dois dígitos)

\footnotetext{
${ }^{1}$ Em muitas situações-problema propostas para crianças, no âmbito escolar, para quantificar é necessário associar um número a uma determinada grandeza, por meio de contagem ou medição. Nesta direção, é requerido o entendimento das relações envolvidas em situações do campo multiplicativo que abarcam a compreensão da grandeza e das suas medidas. Assim, para essas situações-problema dizemos que as grandezas são discretas quando originárias de uma contagem realizada através de números naturais, em que a medida obtida é sempre por uma quantidade expressa por um inteiro (sem fracionamento). Por exemplo: Na sala de aula há quatro estudantes em pé. Por outro lado, as grandezas contínuas são determinadas não apenas por uma contagem, mas também pela aferição de uma medição, sendo expressas por inteiros, fracionários ou decimais, através de números racionais. Por exemplo: A temperatura registrada hoje em Porto Alegre foi de $3,5^{\circ} \mathrm{C}$. Maiores esclarecimentos sobre essas discussões são apresentados por Morais e Teles (2014).
} 
ou grandes (de três ou mais dígitos) surgem como um dos aspectos mais investigados na literatura, sobretudo em pesquisas sobre o conceito de proporção, como é o caso do estudo clássico de Piaget, Grize, Szeminska e Bang (1968) cujo principal resultado foi que as crianças tinham um melhor desempenho em tarefas com quantidades contínuas do que com quantidades discretas. Resultado semelhante foi obtido em diversas pesquisas (BEGOLLI et al., 2020; BOYER; LEVINE, 2015; HURST; CORDES, 2018; JEONG; LEVINE; HUTTENLOCHER, 2007; SPINILLO; BRYANT, 1999) que, de modo geral, revelaram que crianças da Educação Infantil e de Anos Iniciais do Ensino Fundamental apresentavam mais dificuldades em lidar com a proporção quando as quantidades eram discretas do que quando as quantidades eram contínuas.

A explicação dada pelos pesquisadores é que com as quantidades discretas as crianças tendem a usar estratégias de contagem de elementos, gerando respostas incorretas. Por outro lado, o maior sucesso com as quantidades contínuas decorre do fato de raciocinarem em termos relativos e não em termos absolutos. Mix, Huttenlocher e Levine (2002) sugerem que as quantidades contínuas facilitam o raciocínio proporcional porque permitem o uso de pontos de referência de natureza perceptual. Este comentário se aplica, por exemplo, ao estudo conduzido por Spinillo e Bryant (1991) em que crianças desde os 6 anos resolviam com sucesso tarefas de proporção em que podiam usar o referencial de 'metade'.

Igualmente relevante é a influência dos suportes de representação no desempenho e nas estratégias adotadas por crianças na resolução de problemas multiplicativos. Os suportes de representação usualmente investigados são material concreto manipulativo como dedos, fichas, objetos (jarros, flores); recursos gráficos como desenhos, diagramas, gráficos, tabelas; ou mesmo recursos tecnológicos (calculadora, software). Inúmeras são as pesquisas em que se comparam diferentes situações de resolução de problemas que variam em função do suporte de representação disponibilizado.

Selva (1998), por exemplo, comparou o desempenho e as estratégias adotadas por crianças de 6 a 8 anos ao resolverem problemas de divisão inexata por meio de diferentes suportes de representação: lápis e papel, material concreto e cálculo oral. Os dados mostraram que o uso de fichas e de lápis e papel levava a um desempenho semelhante, porém, superior ao que foi obtido na situação sem material, indicando que a situação gráfica favorecia o desempenho da mesma forma que o material concreto. As representações gráficas produzidas com lápis e papel propiciavam estratégias mais flexíveis e apropriadas do que a manipulação das fichas. Enquanto as fichas levavam as crianças a fazerem uma representação direta do enunciado, as representações gráficas favoreciam o uso de estratégias que contemplavam os 
elementos do problema e suas relações.

O efeito da combinação de diferentes suportes de representação na resolução de problemas de divisão inexata foi examinado por Selva e Borba (2005). Três situações foram propiciadas às crianças de 9 a 11 anos de idade: uma em que usavam primeiro papel e lápis e depois calculadora, outra situação em que ocorria o oposto e uma terceira situação em que as crianças usavam manipulativos e depois papel e lápis. A situação que mais auxiliou as crianças a superar as dificuldades com o resto foi aquela em que primeiro usavam lápis e papel e depois a calculadora, passando a compreenderem o número decimal gerado pela calculadora. As autoras concluíram que a combinação e a ordem em que esses suportes são disponibilizados têm um impacto sobre a resolução dos problemas.

Batista e Spinillo (2008) testaram a hipótese de que material concreto definido (aquele com uma relação clara com os referentes das quantidades presentes no enunciado dos problemas como jarros e flores) facilitaria a resolução de problemas de multiplicação e de divisão mais do que material concreto indefinido, cuja relação com os referentes não é evidente (fichas plásticas). A hipótese foi confirmada, uma vez que o material concreto definido favoreceu o desempenho e o uso de procedimentos apropriados pelas crianças de 8 anos, o mesmo não ocorrendo com o material concreto indefinido. A conclusão foi que nem todo material concreto tem o mesmo efeito sobre a resolução de problemas, sendo necessário considerar, além de seu caráter manipulativo, sua relação com os referentes das quantidades presentes no enunciado.

Borba e Pessoa (2013), analisando a literatura na área de resolução de problemas de combinatória, discutem resultados positivos obtidos em pesquisas com crianças. Um dos estudos relatados foi o de Borba e Azevedo (2012) que consistia em comparar o desempenho de crianças do $5^{\circ}$ ano do Ensino Fundamental em diferentes situações de resolução de problemas de combinatória de diferentes tipos. As situações que se mostraram mais proveitosas foram aquelas em que a criança resolvia os problemas por meio da elaboração de árvores de possibilidades com o suporte de um software ou com o suporte de lápis e papel. Os principais avanços das crianças ao usarem a árvore de possibilidades foram em relação ao controle e monitoramento das combinações. Este gerenciamento do processo de resolução evitava que fossem repetidas as combinações já feitas e permitia, ainda, que fossem estabelecidas todas as combinações possíveis. As autoras concluíram que a elaboração de árvores de possibilidades, sejam virtuais ou gráficas, é um recurso facilitador do desempenho na resolução de problemas de combinatória, especialmente os de produto cartesiano.

O papel facilitador da listagem e, sobretudo, da árvore de possibilidades como suportes de representação foram apontados por Azevedo, Borba e Bittar (2020) com estudantes de 11 a 
13 anos. Nesta investigação foram analisadas as diferentes maneiras usadas por estudantes do Ensino Fundamental para identificar e realizar conversões entre diferentes modalidades de registros em situações combinatórias e, em seguida, foi conduzida uma intervenção com vistas a auxiliar as crianças a superarem as dificuldades que haviam sido inicialmente identificadas. A intervenção que se mostrou a mais favorável foi aquela em que foi explorada a árvore de possibilidades como suporte intermediário para auxiliar nas conversões da linguagem natural para a linguagem matemática (expressões numéricas).

Tomados de forma conjunta, os dados dessas pesquisas revelam que tanto as características numéricas dos problemas como os suportes de representação disponibilizados são fatores que influenciam a resolução de problemas que envolvem conceitos de natureza multiplicativa. Todavia, além desses, há um outro aspecto que está intrinsecamente associado ao raciocínio necessário para a resolução de problemas de natureza multiplicativa: as relações uma para muitos.

De acordo com Vergnaud (1983; 1997; 2003), a relação um para muitos, também denominada correspondência um para muitos, é inerente ao raciocínio necessário para a resolução de problemas que envolvem conceitos que estão inseridos no campo conceitual das estruturas multiplicativas como a divisão, a multiplicação e conceitos relacionais como fração, proporção, porcentagem, probabilidade e combinatória. A correspondência uma para muitos se caracteriza pela relação entre conjuntos disjuntos, em que um dado elemento de um conjunto se relaciona com elementos de outro conjunto, por exemplo, um carro para quatro rodas. No caso de haver três carros haverá 12 rodas, de modo que a razão (1:4) se mantém constante, mesmo quando a quantidade de elementos varia (3:12). A correspondência um para muitos envolve a compreensão do conceito de proporção e fator escalar, como discutem Nunes e Bryant (1996) e Gitirana et al. (2014).

É importante enfatizar que o estabelecimento da razão é aspecto crucial na resolução de problemas de estrutura multiplicativa. Na realidade, é o estabelecimento de relações que constitui o pensamento multiplicativo, e a razão é, em última instância, um tipo de relação que é usada como uma estratégia de resolução (AVCU; DOĞAN, 2014), uma relação um para muitos.

A ideia é a de que, da mesma forma que nas pesquisas são controladas as características numéricas dos problemas e os suportes de representação disponibilizados, é possível, também, controlar a maneira como as relações um para muitos são apresentadas nos problemas de estrutura multiplicativa. Retomando a questão acerca de quais situações seriam mais fáceis para as crianças resolverem problemas de estrutura multiplicativa, é possível supor que seriam 
aquelas em que a correspondência um para muitos estaria explicitamente mencionada no enunciado do problema. Esta ideia encontra respaldo em pesquisas sobre o raciocínio combinatório, como comentado a seguir.

De acordo com Nunes e Bryant (1996), uma das dificuldades que as crianças enfrentam ao resolver problemas de produto cartesiano reside no fato de que a correspondência um para muitos está implícita. Para examinar esta possibilidade, pesquisas foram realizadas em que crianças eram apresentadas a situações em que essa correspondência era explicitamente mencionada, observando-se o impacto positivo da explicitação das relações uma para muitos na resolução de problemas de produto cartesiano (MELO; SILVA; SPINILLO, 2016; SILVA; SPINILLO, 2020; SPINILLO; SILVA, 2010).

No estudo conduzido por Spinillo e Silva (2010), crianças de 7-8 anos resolveram problemas de produto cartesiano em duas situações distintas. $\mathrm{Na}$ situação 1, os princípios invariantes que governam o raciocínio combinatório, incluindo a correspondência uma para muitos, eram apresentados de maneira implícita, como usualmente proposto nas pesquisas na área. $\mathrm{Na}$ situação 2, esses princípios eram explicitamente mencionados no enunciado dos problemas. O desempenho foi significativamente melhor na situação explícita do que na implícita, tanto em relação ao número de acertos como em relação às estratégias adotadas na resolução dos problemas. Os dados revelaram ainda que o desempenho na situação em que as relações eram implícitas melhorava significativamente quando os problemas eram resolvidos após a situação em que as relações eram explícitas. A conclusão foi que as crianças são capazes de resolver problemas de produto cartesiano quando os princípios invariantes são explicitamente mencionados e que, também, são capazes de aplicar esses princípios na resolução de problemas em que esses princípios estão implícitos.

Em estudo subsequente, Melo, Silva e Spinillo (2016) investigaram se a explicitação dos princípios invariantes do raciocínio combinatório, incluindo a relação um para muitos, também se aplicaria à resolução de problemas de combinação. Os dados revelaram que o papel facilitador da explicitação da relação um para muitos era mais efetivo na resolução de problemas de produto cartesiano, mas não era expressivo na resolução de problemas de combinação que envolviam subgrupos de elementos.

Silva e Spinillo (2020) discutem resultados de pesquisas que buscam alternativas para desenvolver formas apropriadas de resolução de problemas que envolvem o raciocínio combinatório, especificamente problemas de produto cartesiano. A partir desta discussão, as autoras ressaltam a importância da explicitação das relações um para muitos no raciocínio combinatório, seja por meio de diagramas ou por meio da linguagem. Nesta análise, são 
destacados os estudos em que a árvore de possibilidades é apontada como um suporte de representação que facilita a resolução de problemas de combinatória, como documentado por Borba e Azevedo (2012) e por Borba e Pessoa (2013) em que o diagrama típico da árvore de possibilidades deixa explícita a correspondência um para muitos. O uso de setas e traços que ligam um elemento de um conjunto a outros de outro conjunto permite que as crianças gerenciem as diferentes relações um para muitos que se estabelecem neste tipo de problema e, assim, avancem nos procedimentos de resolução. Na realidade, o papel facilitador da árvore de possibilidades reside no fato de que este diagrama permite que as relações um para muitos sejam graficamente explicitadas.

A partir dessa discussão, é possível supor que explicitar a correspondência um para muitos possa também melhorar o desempenho das crianças em problemas de multiplicação e divisão. Esta possibilidade é explorada nesta investigação. Uma vez que a relação um para muitos é essencial no raciocínio multiplicativo, é importante examinar se a maneira como esta relação é expressa no enunciado dos problemas teria um efeito sobre o desempenho de crianças. Considerando a sugestão de Nunes e Bryant (1996) e os dados obtidos nos estudos sobre o raciocínio combinatório realizados por Silva e Spinillo (2020) e Spinillo e Silva (2010) examinou-se a possibilidade de que o nível de explicitação da relação um para muitos seria fator importante na resolução de problemas multiplicativos e que, quanto mais explícitas forem essas relações, melhor seria o desempenho das crianças. Para isto, no presente estudo, as relações um para muitos, no enunciado dos problemas, variavam quanto ao seu nível de explicitação.

\section{Método}

\subsection{Participantes}

Participaram do estudo 1160 estudantes de ambos os sexos, divididos em dois grupos: 591 alunos do $3^{\circ}$ ano (com idades entre 8 e 9 anos) e 569 alunos do $5^{\circ}$ ano do Ensino Fundamental (com idades entre 10 e 11 anos) de escolas públicas localizadas nas cidades de Recife (PE), Ilhéus (BA) e Fortaleza (CE).

\subsection{Procedimento e planejamento experimental}

Os participantes foram solicitados a resolver, por escrito e individualmente, três 
problemas $^{2}$ apresentados em uma ordem fixa (P1, P2 e P3), um por página, com espaço para a resolução e registro da resposta, em páginas sequenciadas em formato de um livro. A aplicação foi feita coletivamente em sala de aula. $\mathrm{O}$ examinador lia o enunciado de cada problema e solicitava que os alunos o resolvesse, um por vez, disponibilizando lápis e borracha. O examinador fez a leitura em voz alta dos problemas com o objetivo de evitar eventuais dificuldades de leitura apresentadas pelos alunos. Apenas quando todos os estudantes haviam concluído a resolução de um dado problema era feita a leitura do enunciado do problema seguinte e iniciada sua resolução.

Os problemas se assemelhavam aos problemas encontrados usualmente em livros didáticos, sendo descritos a seguir:

Problema 1: Joana sabe que em um pacote há 6 biscoitos. Ela tem 5 pacotes.Quantos biscoitos Joana tem?

Problema 2: A Escola Recanto fará uma festa para 36 convidados. Em cada mesa ficarão 4 convidados. Quantas mesas a escola precisará alugar?

Problema 3: Um supermercado fez uma promoção: "Leve 4 litros de suco por apenas 12 reais". Quanto vai custar cada litro de suco? Fonte: Dados Pesquisa Rede E-Mult

Os problemas variavam conforme o grau de explicitação das relações um para muitos que apresentavam. No Problema 1, a relação um para muitos (um para seis) era explicitamente mencionada logo na primeira frase do enunciado ("Joana sabe que em um pacote há seis biscoitos"). No Problema 2, a relação (um para quatro) era explicitada na segunda frase do enunciado ("Em cada mesa ficarão quatro convidados"). No Problema 3 a relação um para muitos não era explicitada, pois a razão consistia em quatro litros de suco para 12 reais, tendo que ser encontrada (um para três).

Tanto no Problema 1 como no Problema 2, o fator unitário era literalmente explicitado no enunciado, podendo servir de base para a resolução dos problemas. No Problema 3, por outro lado, o fator unitário não era explicitamente mencionado, tendo que ser inferido. Assim, nos três problemas, o grau de explicitação das relações um para muitos variava, de modo que no Problema 1 era de alta explicitação, no Problema 2 era de média explicitação e no Problema 3 esta relação era implícita. A predição era que a de que o desempenho das crianças seria melhor no Problema 1 e no Problema 2, apresentando dificuldades no Problema 3.

\footnotetext{
${ }^{2}$ Os problemas fizeram parte de um instrumento composto por 14 problemas utilizado no projeto intitulado "Um domínio das estruturas multiplicativas no Ensino Fundamental” (No. 15727, OBEDUC - Observatório da Educação) referente ao Edital 049/2012/CAPES/INEP.
} 


\section{Resultados}

Os dados foram analisados em função do número de acertos obtidos em cada problema (total de 3.480 problemas), sendo as médias de respostas corretas e outros dados estatísticos apresentados na Tabela 1.

Tabela 1 - Desempenho médio dos estudantes por problema e ano escolar

\begin{tabular}{|c|c|c|c|c|c|c|c|c|}
\hline \multirow[b]{2}{*}{ Problemas } & \multirow[b]{2}{*}{ Ano } & \multirow[b]{2}{*}{$\begin{array}{l}\mathrm{N}^{0} \text { de } \\
\text { estudantes }\end{array}$} & \multirow[b]{2}{*}{ Média } & \multirow[b]{2}{*}{$\begin{array}{l}\text { Desvio } \\
\text { padrão }\end{array}$} & \multicolumn{2}{|c|}{$\begin{array}{c}\text { Intervalo de } \\
\text { confiança para média }\end{array}$} & \multirow[b]{2}{*}{ Mínimo } & \multirow[b]{2}{*}{ Máximo } \\
\hline & & & & & $\begin{array}{c}\text { confianç } \\
\text { Limite } \\
\text { inferior }\end{array}$ & $\begin{array}{l}\text { ara média } \\
\text { Limite } \\
\text { superior }\end{array}$ & & \\
\hline \multirow[t]{2}{*}{ P1 } & $3^{\circ}$ & 591 & ,39 & ,488 &, 35 &, 43 & 0 & 1 \\
\hline & $5^{\circ}$ & 569 & 68 & 466 & 64 & ,72 & 0 & 1 \\
\hline \multirow[t]{2}{*}{ P2 } & $3^{\circ}$ & 591 & ,26 & 439 & ,23 & ,30 & 0 & 1 \\
\hline & $5^{\circ}$ & 569 & ,41 & 492 & ,37 & ,45 & 0 & 1 \\
\hline \multirow[t]{2}{*}{ P3 } & $3^{\circ}$ & 591 & ,19 & ,390 & ,15 & 22 & 0 & 1 \\
\hline & $5^{\circ}$ & 569 & ,40 & , 490 & ,36 & ,44 & 0 & 1 \\
\hline
\end{tabular}

Nota: P1: alta explicitação; P2: média explicitação; P3: sem explicitação Fonte: Dados Pesquisa Rede E-Mult

O teste McNemar para amostras emparelhadas detectou diferenças significativas entre os problemas tanto no $3^{\circ}$ ano como no $5^{\circ}$ ano, como mostram os valores de significância na Tabela 2.

Tabela 2 - Valores de significância do teste McNemar

\begin{tabular}{|c|c|c|}
\hline $\begin{array}{l}\text { Comparações entre } \\
\text { problemas }\end{array}$ & $3^{\circ}$ ano & $5^{\circ}$ ano \\
\hline P1 vs P2 & $\mathrm{X}^{2}=74,052 ; \mathrm{gl}=1 ; \mathrm{p}=.000$ & $\mathrm{X}^{2}=45,436 ; \mathrm{gl}=1 ; \mathrm{p}=.000$ \\
\hline P1 vs. P3 & $\mathrm{X}^{2}=82,778 ; \mathrm{gl}=1 ; \mathrm{p}=.000$ & $X^{2}=39,542 ; g l=1 ; p=.000$ \\
\hline P2 vs. P3 & $X^{2}=53,353 ; g l=1 ; p=.000$ & $\mathrm{X}^{2}=68,887 ; \mathrm{gl}=1 ; \mathrm{p}=.000$ \\
\hline
\end{tabular}

Nota: P1: alta explicitação; P2: média explicitação; P3: sem explicitação.

Fonte: Dados Pesquisa Rede E-Mult

O teste revelou que o desempenho variou entre os três problemas, pois as crianças foram mais bem-sucedidas no Problema 1 e no Problema 2 do que no Problema 3. Isso ocorreu porque, como mencionado, nos dois primeiros problemas a correspondência um para muitos era explicitada no enunciado, enquanto no Problema 3 essa relação estava implícita, tendo que ser buscada. Os dados mostraram que o Problema 1 era ainda mais fácil que o Problema 2, dado que naquele a relação um para muitos era ainda mais explícita do que no Problema 2, por ser mencionada logo na primeira frase do enunciado. Este padrão de resultados ocorreu em relação aos dois grupos de participantes (Tabela 3). 
Tabela 3 - Número e percentual (entre parênteses) de respostas corretas em cada problema

\begin{tabular}{cccc}
\multicolumn{4}{c}{ por ano escolar } \\
\hline Ano & $\begin{array}{c}\text { Problema 1 } \\
\text { (alta explicitação) }\end{array}$ & $\begin{array}{c}\text { Problema 2 } \\
\text { (média explicitação) }\end{array}$ & $\begin{array}{c}\text { Problema 3 } \\
\text { (sem explicitação) }\end{array}$ \\
\hline $3^{\circ}$ & 231 & 154 & 110 \\
$(\mathrm{n}=495)$ & $(46,7)$ & $(31,1)$ & $(22,2)$ \\
$5^{\circ}$ & 388 & 232 & 227 \\
$(\mathrm{n}=847)$ & $(45,8)$ & $(27,4)$ & $(26,8)$ \\
\hline
\end{tabular}

Fonte: Dados Pesquisa Rede E-Mult

De acordo com os resultados, independentemente do ano escolar, as crianças apresentam um bom desempenho em problemas em que a relação um para muitos é explicitamente mencionada no enunciado, sobretudo quando ela consta logo na primeira frase do enunciado. Este dado será discutido nas conclusões deste estudo.

O Teste Qui-quadrado detectou diferenças significativas entre os anos escolares nos três problemas: Problema 1 (alta explicitação: $\mathrm{X}^{2}=98,664$; $\mathrm{gl}=1 ; \mathrm{p}=.000$ ); Problema 2 (média explicitação $\mathrm{X}^{2}=28,274 ; \mathrm{gl}=1 ; \mathrm{p}=.000$ ); e Problema 3 (sem explicitação: $\mathrm{X}^{2}=63,702 ; \mathrm{gl}=1$; $\mathrm{p}=.000$ ). Verificou-se que as crianças do $5^{\circ}$ ano tiveram sistematicamente um desempenho melhor que as do $3^{\circ}$ ano em cada um dos problemas.

A seguir são apresentados alguns exemplos que ilustram a maneira como as crianças lidavam de maneira apropriada com as relações um para muitos nos problemas em que obtiveram um melhor desempenho (Problema 1 e Problema 2):

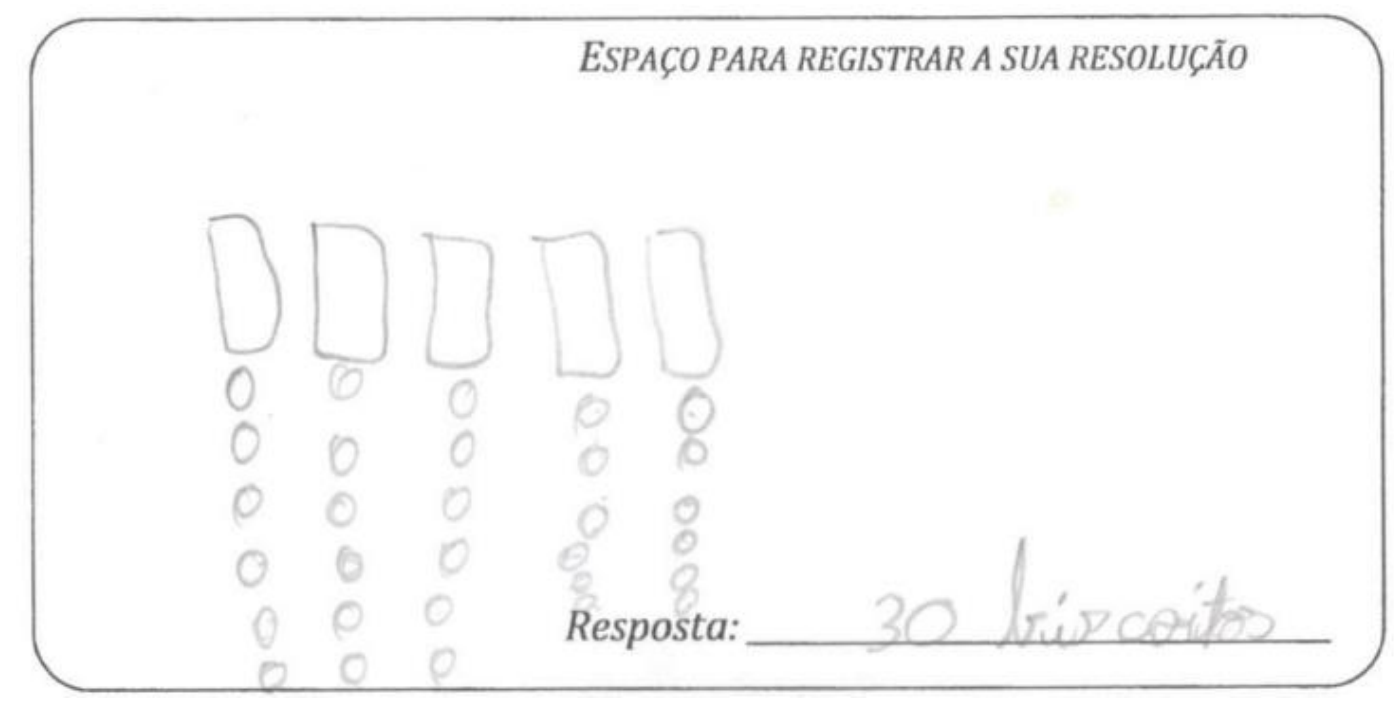

Figura 1 - Exemplo: Problema 1, aluno do $3^{\circ}$ ano Fonte: Dados Pesquisa Rede E-Mult

Neste exemplo, a criança desenha cinco pacotes (retângulos) e os associa a seis biscoitos (pequenos círculos), deixando clara a correspondência um para muitos (um pacote para seis biscoitos), fornecendo o resultado apropriado, no caso, "30 biscoitos", como pode ser visto no espaço reservado para que a criança escrevesse sua resposta. 


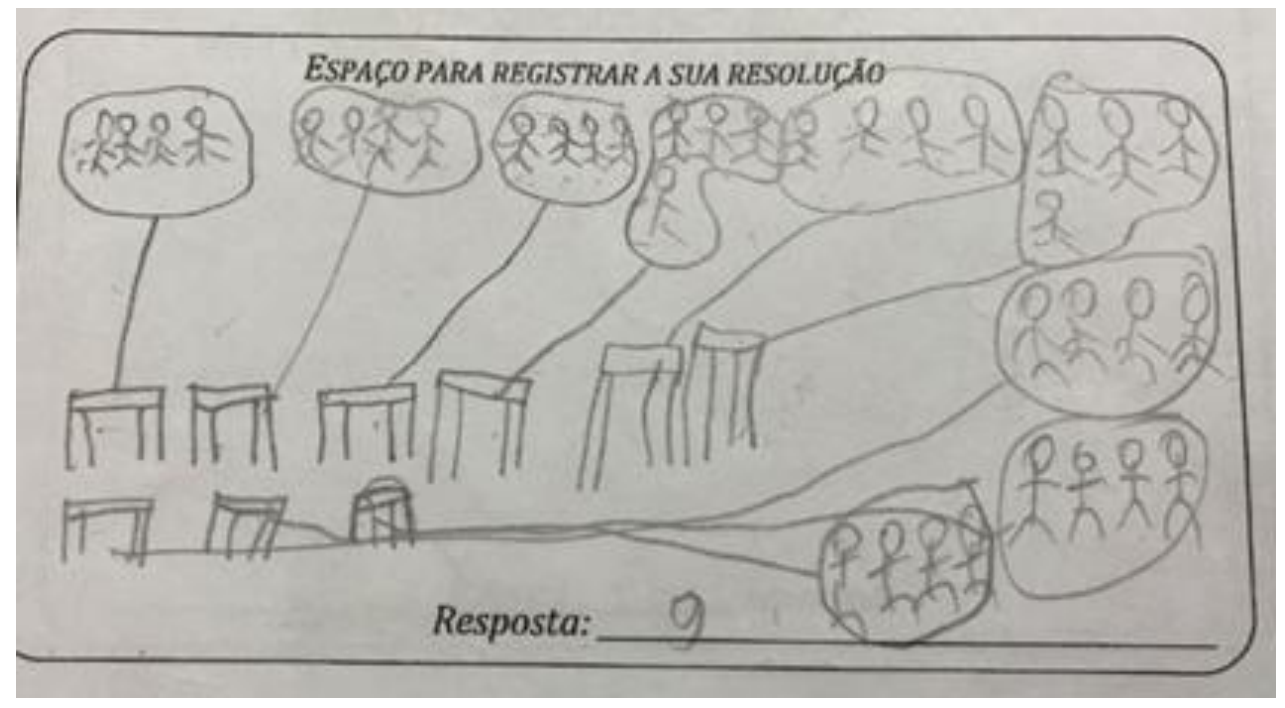

Figura 2 - Exemplo: Problema 2, aluno do $3^{\circ}$ ano

Fonte: Dados Pesquisa Rede E-Mult

Na representação feita pela criança é claramente estabelecida a correspondência um para muitos, no caso, uma mesa para quatro pessoas. Para isso o participante agrupou as 36 pessoas em grupos de quatro por meio de um círculo em torno das pessoas; e, por meio de um traço, associou cada grupo de quatro pessoas a uma mesa. Contou, então, quantos grupos havia, chegando ao resultado 'nove', como indicado no espaço dedicado à resposta.

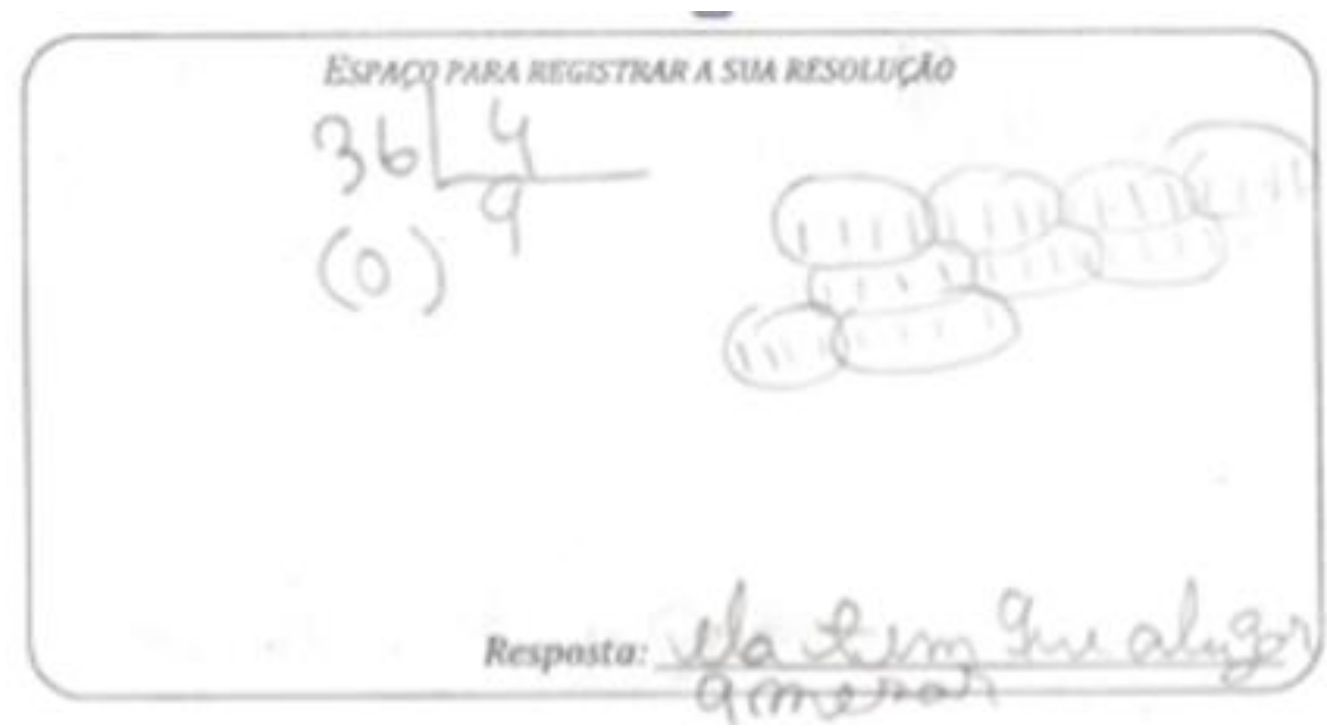

Figura 3 - Exemplo: Problema 2, aluno do $5^{\circ}$ ano Fonte: Dados Pesquisa Rede E-Mult

Neste exemplo, a criança optou por adotar dois tipos de representação: simbólica e icônica. A representação simbólica consistiu no uso do algoritmo da operação de divisão, aquele amplamente aceito no contexto escolar, usando os simbolismos típicos da operação armada da divisão. A representação icônica, por sua vez, consistiu no uso de tracinhos que representavam pessoas que eram agrupadas de quatro em quatro por meio de uma linha que circulava os 
tracinhos que representavam as pessoas. Entende-se que cada agrupamento (circulado pela linha) correspondia a uma mesa que, diferentemente do observado no exemplo anterior, não foi desenhada. Semelhante ao que foi observado no Exemplo 2, a criança contou quantos agrupamentos havia, chegando ao resultado nove, que foi registrado por meio da resposta "ela tem que alugar 9 mesas".

Como discutido adiante, devido à aplicação ter ocorrido de forma coletiva, não foi possível obter-se detalhes acerca da maneira como a criança havia chegado ao resultado dos problemas. No Exemplo 1, não é possível saber se após estabelecer as relações um para muitos a criança procedeu a uma adição ou a uma multiplicação. No Exemplo 2, por sua vez, não é possível saber se a criança foi desenhando cada grupo de quatro pessoas e simultaneamente foi contando até chegar ao total de 36 pessoas; ou se o desenho feito foi a representação final da resolução empregada. Outras estratégias poderiam ter sido adotadas como, por exemplo, o aluno ter desenhado todas as mesas e associado quatro pessoas em cada até chegar em 36 pessoas. Comentário semelhante pode ser feito acerca do Exemplo 3, pois não se sabe se primeiro a criança utilizou o algoritmo da divisão e em seguida fez os agrupamentos, ou se ocorreu o contrário.

Uma compreensão mais detalhada dos procedimentos adotados pelos participantes só seria possível caso a pesquisa fosse feita de uma entrevista do tipo clínico-crítico em que o examinador acompanhasse o raciocínio das crianças no momento da resolução dos problemas, inclusive, fazendo perguntas de esclarecimento. Esta questão será discutida adiante, como um ponto a ser considerado em pesquisa futura.

\section{Conclusões e discussão}

De modo geral, a questão que norteou a presente investigação foi: que situações podem favorecer a resolução de problemas de estrutura multiplicativa por crianças? A maioria das pesquisas que direta ou indiretamente esclarecem esta questão aponta que a natureza das quantidades, ou seja, as características numéricas do problema, é fator importante, mostrando que o desempenho das crianças é melhor em situações em que os problemas envolvem quantidades contínuas do que quando as quantidades são discretas. Outras investigações, por sua vez, evidenciam que o suporte de representação é fator importante, e que o material concreto que traz uma clara associação com os referentes do enunciado dos problemas parece facilitar o desempenho.

Entretanto, além das características numéricas e dos suportes de representação, outro 
fator é apontado como relevante na resolução de problemas de estrutura multiplicativa: o estabelecimento da razão. Como discutido, a razão está associada à relação um para muitos que é inerente a diversos conceitos inseridos no campo das estruturas multiplicativas. Em problemas que envolvem esses conceitos, a correspondência um para muitos pode estar expressa de maneira mais evidente ou menos. Contudo, embora sejam inúmeras as pesquisas que controlam as situações de resolução de problemas quanto às características numéricas e quanto aos suportes de representação disponibilizados, a explicitação das relações uma para muitos no enunciado dos problemas não tem sido metodologicamente considerada nessas investigações.

Em vista disso, na presente pesquisa foi examinada a possibilidade de que problemas de estrutura multiplicativa de multiplicação e de divisão seriam mais fáceis de serem resolvidos quando tais relações fossem explicitamente mencionadas no enunciado. Para isso, três problemas com diferentes graus de explicitação das relações um para muitos foram solucionados por crianças do $3^{\circ}$ e $5^{\circ}$ ano do Ensino Fundamental. O principal resultado foi que, como esperado, o desempenho foi melhor nos problemas em que a correspondência um para muitos era explicitamente mencionada no enunciado do problema, sendo isso observado em ambos os anos escolares.

Também foi feita a predição de que o Problema 1 seria mais fácil que o Problema 2, dado que nele a correspondência um para muitos era ainda mais explícita do que no Problema 2 por ser mencionada logo na primeira frase do enunciado. De fato, isso ocorreu; porém é relevante comentar que o Problema 1 requeria a operação de multiplicação para sua solução, enquanto o Problema 2 envolvia a divisão. É possível pensar que o melhor desempenho no Problema 1 decorreu também do fato de a multiplicação ser uma operação mais fácil que a divisão (dada sua relação com a adição de parcelas repetidas). Contudo, o uso da operação de multiplicação não pode ser entendido como o fator determinante do desempenho, pois o sucesso dos estudantes foi mais expressivo no Problema 2 do que no Problema 3, apesar de ambos envolverem a operação de divisão. Isso sugere que o nível de explicitação das relações um para muitos, que era mais alto no Problema 2 que no Problema 3, tenha sido o fator determinante.

Por se tratar de um estudo exploratório, é necessário que em pesquisas futuras esses aspectos sejam controlados em um instrumento elaborado especificamente para este fim em que o tipo de operação (divisão e multiplicação) varie em cada um dos três níveis de explicitação (alta, média e baixa). Um estudo com este tipo de planejamento experimental poderia esclarecer qual fator seria mais determinante do desempenho, ou se a combinação entre eles seria a situação mais proveitosa.

É relevante tecer algumas considerações acerca dos procedimentos adotados pelos 
participantes na resolução dos problemas, como aqueles ilustrados nos exemplos apresentados. Cabe ressaltar que os resultados desta investigação foram obtidos por meio de uma aplicação coletiva em sala de aula, o que não permitiu ao examinador interagir com as crianças de modo a obter informações detalhadas sobre suas formas de lidar com as relações um para muitos em cada problema. Assim, outro aspecto a ser considerado em pesquisas futuras seria coletar os dados por meio de uma entrevista clínico-crítica em que o examinador pudesse, individualmente, endereçar perguntas que esclarecessem os procedimentos de resolução adotados em cada problema.

Apesar de exploratório, o estudo corrobora resultados obtidos em pesquisas anteriores que apontam o papel facilitador que a explicitação da correspondência um para muitos desempenha na resolução de problemas de estrutura multiplicativa, adicionando mais evidências a favor da explicitação dessas relações. Esta contribuição de natureza teórica tem implicações aplicadas de natureza educacional. Por exemplo, poderiam ser elaboradas atividades didáticas em que a explicitação da razão levasse os alunos a perceberem que a relação um para muitos é um modo de raciocinar que facilita a compreensão de situações que envolvem conceitos próprios das estruturas multiplicativas, inclusive aqueles mais complexos como a proporção e a combinatória.

Nesta direção, a razão unitária assume papel importante em situações de ensino de conceitos multiplicativos, como evidenciado em estudos de intervenção (FIELDING-WELLS; DOLE; MAKAR, 2014; PARISH, 2010; ŞEN; GÜLER, 2017). Um exemplo, são os resultados positivos obtidos por Şen e Güler (2017) em que o uso da razão unitária (1:X) era uma estratégia intuitiva que os estudantes usavam para raciocinar proporcionalmente de maneira cada vez mais elaborada. De modo geral, essas investigações mostram que estratégias didáticas proveitosas podem ser aquelas que envolvem a instrução direta sobre a razão unitária. Assim, torna-se relevante conduzir estudos de intervenção explorando a razão unitária e a razão não unitária em problemas envolvendo conceitos matemáticos mais simples e mais complexos. Na realidade, a instrução explícita de estratégias apropriadas tem beneficiado crianças com dificuldades de aprendizagem em Matemática (GERSTEN et al., 2009; ZHANG et al., 2014).

Para finalizar, parece existir um campo profícuo de investigação acerca do papel desempenhado pela explicitação das relações um para muitos na compreensão da criança sobre conceitos matemáticos de natureza multiplicativa. Compreendido este papel, situações didáticas poderão ser elaboradas e propostas em salas de aula do Ensino Fundamental. 


\section{Agradecimentos}

Agradecemos o apoio financeiro recebido da Coordenação de Aperfeiçoamento de Pessoal de Nível Superior (CAPES) para o desenvolvimento da pesquisa realizada no âmbito do Programa Observatório da Educação (OBEDUC), referente ao Edital 049/2012/CAPES/INEP; e à Fundação de Amparo à Ciência e Tecnologia do Estado de Pernambuco (FACEPE) que concedeu bolsas de Iniciação Científica e de Apoio Técnico para a realização da investigação na cidade do Recife.

\section{Referências}

AVCU, R.; DOĞAN, M. What are the strategies used by seventh grade students while solving proportional reasoning problems? International Journal of Educational Studies in Mathematics, Ancara, v. 1, n. 2, p. 34-55, 2014.

AZEVEDO, J.; BORBA, R. E. S. R.; BITTAR, M. Intermediate representations in the learning of combinatorial situations. Educação e Realidade, Porto Alegre, v. 1, p. 1-26, 2020.

BATISTA, A. M. B.; SPINILLO, A. G. Nem todo material concreto é igual: a importância dos referentes na resolução de problemas. Estudos de Psicologia, Natal, v. 13, n. 1, p. 13-21, 2008.

BEGOLLI, K. N.; BOOTH, J. L.; HOLMES, C. A.; NEWCOMBE, N. S. How many apples make a quarter? The challenge of discrete proportional formats. Journal of Experimental Child Psychology, Amsterdã, v. 192, p. 32-47, 2020.

BORBA, R. E. R.; AZEVEDO, J. A construção de árvores de possibilidades com recurso computacional: o desenvolvimento do raciocínio combinatório de Karine e Vitória. In: SPINILLO, A. G.; LAUTERT, S. L. (ed.). A pesquisa em Psicologia e suas implicações para a Educação Matemática. Recife: Editora Universitária da UFPE, 2012. p. 89-138.

BORBA, R. E. S.; PESSOA, C. A. Estudos em raciocínio combinatório. In: BORBA, R. E. S.; MONTEIRO, C. E. Monteiro (ed.). Processos de ensino e aprendizagem em Educação Matemática. Recife: Editora Universitária da UFPE, 2013. p. 11-54.

BOYER, T. W.; LEVINE, S. C. Prompting children to reason proportionally: Processing discrete units as continuous amounts. Developmental Psychology, Washington, v. 51, n. 5, p. 615-620, 2015.

FIELDING-WELLS, J.; DOLE, S.; MAKAR, K. Inquiry pedagogy to promote emerging proportional reasoning in primary students. Mathematics Education Research Journal, Queensland, v. 26, n. 1, p. 47-77, 2014.

GERSTEN, R.; CHARD, D. J.; JAYANTHI, M.; BAKER, S. K.; MORPHY, P.; FLOJO, J.

Mathematics instruction for students with learning disabilities: A meta-analysis of instructional components. Review of Educational Research, Newbury Park, v. 79, n. 3, p. 1202-1242, 2009.

GITIRANA, V., CAMPOS, T. M. M., MAGINA, S. P.; SPINILLO, A. G. Repensando multiplicação e divisão: Contribuições da teoria dos campos conceituais. São Paulo: Editora PROEM, 2014.

HURST, M. A.; CORDES, S. Attending to relations: Proportional reasoning in 3- to 6-year-old 
children. Developmental Psychology, Washington, v. 54, n. 3, p. 428-439, 2018.

JEONG, Y., LEVINE, S. C.; HUTTENLOCHER, J. The development of proportional reasoning: Effect of continuous versus discrete quantities. Journal of Cognition and Development, London, v. 8, n. 2, p. 237-256, 2007.

MELO, L. M. S.; SILVA, J. F. G da; SPINILLO, A. G. Os princípios invariantes e a resolução de problemas de raciocínio combinatório. Em Teia - Revista de Educação Matemática e Tecnológica Iberoamericana, Recife, v. 7, n. 1, p. 1-120, 2016.

MIX, K. L.; HUTTENLOCHER, J.; LEVINE, S. C. Multiple cues for quantification in infancy: Is number one of them? Psychological Bulletin, Washington, v. 128, n. 2, p. 278-294, 2002.

MORAIS, M. D.; TELES, R. A. de M. Grandezas e medidas no ciclo de alfabetização. Cadernos da TV Escola: Um salto para o futuro. Grandezas e medidas no ciclo de alfabetização. Boletim 24, n. 8, p. 10-16, 2014.

NUNES, T.; BRYANT, P. Children doing mathematics. Oxford: Blackwell, 1996.

PARISH, L. Facilitating the development of proportional reasoning through teaching ratio. In: SPARROW, L.; KISSANE, B.; HURST C. (ed.). Proceedings of the $33^{\text {rd }}$ annual conference of the Mathematics Education Research Group of Australasia. Fremantle: MERGA, 2010. p. 469-476.

PIAGET, J.; GRIZE, J.-B.; SZEMINSKA, A.; BANG, V. Epistemologie et psichologie de la fonction. Paris: Presses Universitaires de France, 1968.

SELVA, A. C. V. Discutindo o uso de materiais concretos na resolução de problemas de divisão. In: SCHLIEMANN, A. D.; CARRAHER, D. W. (ed.). A compreensão de conceitos aritméticos: ensino e pesquisa. Campinas: Papirus, 1998. p. 95-119.

SELVA, A.; BORBA, R. O uso de diferentes representações na resolução de problemas de divisão inexata: analisando a contribuição da calculadora. Boletim GEPEM, Rio de Janeiro, v. 47, p.51-72, 2005 .

ŞEN, C.; GÜLER, G. Effect of strategy teaching for the solution of ratio problems on students' proportional reasoning skills. Malaysian Online Journal of Educational Sciences, Kuala Lumpur, v. 5 , n. 2, p. $1-15,2017$.

SILVA, J. F. G. da; SPINILLO, A. G. Relações diretas e inversas em problemas de produto cartesiano: É possível facilitar a compreensão de crianças a partir da explicitação dos princípios invariantes? In: MARTINS, E.; LAUTERT, S. (ed.), Diálogos sobre o ensino, a aprendizagem e a formação de professores: contribuições da psicologia da educação matemática. Rio de Janeiro: Autografia, 2020, p. 123-154.

SPINILLO, A.G.; BRYANT, P. Children's proportional judgements: the importance of 'half'. Child Development, New York, v. 62, p. 427-440, 1991.

SPINILLO, A.G.; BRYANT, P. Proportional reasoning in young children: part-part comparisons about continuous and discontinuous quantities. Mathematical Cognition, London, v. 5, n. 2, 1999, p. 181197.

SPINILLO, A.G.; SILVA, J. F.G. da. Making explicit the principles governing combinatorial reasoning: does it help children to solve Cartesian product problems? Proceedings of the $34^{\text {rd }}$ Conference of the International Group for the Psychology of Mathematics Education, Belo Horizonte: PME, 2010, p. 216-224. 
VERGNAUD, G. Multiplicative structures. In: LESH, R. A.; LANDAU, M. (ed.). Acquisition of mathematical concepts and processes. New York: Academic Press, 1983. p. 127-174.

VERGNAUD, G. The nature of mathematical concepts. In: NUNES, T.; BRYANT, P. (ed.). Learning and teaching mathematics: An international perspective. Hove: Psychology Press, 1997. p. 5-28.

VERGNAUD, G. A gênese dos campos conceituais. In: GROSSI, E. P. (ed.). Por que ainda há quem não aprende? A teoria. Rio de Janeiro: Vozes, 2003. p. 21-64.

ZHANG, D.; XIN, Y. P.; HARRIS, K.; DING, Y. Improving multiplication strategic development in children with math difficulties. Learning Disability Quarterly, New York, v. 37, n. 1, p. 15-30, 2014.

Submetido em 07 de Abril de 2020. Aprovado em 14 de Agosto de 2020. 